Open Access

\title{
Full-duplex amplify-and-forward relays with optimized transmission power under imperfect transceiver electronics
}

\author{
Gustavo J. González ${ }^{1 *}$, Fernando H. Gregorio' ${ }^{1}$ Juan E. Cousseau' ${ }^{1}$, Taneli Riihonen² and Risto Wichman²
}

\begin{abstract}
In-band full-duplex (FD) relays are useful for extending coverage areas and increasing overall throughput in wireless networks. The main technical difficulty hindering their implementation and use is their inherent self-interference (SI), generated due to simultaneous in-band reception and forwarding. Efficient SI mitigation is a practical necessity, and the imperfections in transceiver electronics, from which power amplifier (PA) non-linearity is one of the most serious phenomena, have to be taken into account in order to not limit the performance of such techniques. The magnitude of the distortion introduced by the PA depends on the relay input back-off (IBO) whose optimization for alleviating the effect of PA non-linearity is the main research objective in this paper. In particular, although plain signal-to-noise ratio (SNR) at the destination obviously increases when the IBO decreases, increased transmit power also strengthens the non-linear distortion leading to decreasing overall signal-to-interference-plus-noise ratio (SINR). We develop expressions for bounding the optimal IBO setting that maximizes the SINR at the destination, considering all relevant hardware impairments and SI cancellation with I/Q imbalance compensation. We provide closed-form solutions for the soft-limiter PA model and numerical results for more general PA models. Finally, the derived IBO bounds are compared with the numerical maximization of the SINR and the minimization of the bit-error rate (BER) to demonstrate that the theoretical bound settings provide good approximations to the optimal one.
\end{abstract}

Keywords: In-band full-duplex wireless communication, Non-regenerative relays, Optimization, Self-interference cancellation, Transceiver hardware impairments

\section{Introduction}

Advances in electronics, integration techniques, and signal processing are about to enable real full-duplex (FD) operation without frequency division in wireless transceivers, which is one of the most sought objectives since the invention of radio transmissions [1]. In contrast to conventional half-duplex (HD) transceivers, a FD transceiver is able to transmit and receive simultaneously in the same frequency band. Besides doubling the theoretical spectral efficiency, this may open up a wide range of new applications from cognitive radio spectral sensing [2] to more efficient medium access protocols [3-5]. This

*Correspondence: ggonzalez@uns.edu.ar

${ }^{1}$ CONICET-Department of Electrical and Computer Engineering, Universidad Nacional del Sur, Av. Alem 1253, Bahía Blanca, Argentina

Full list of author information is available at the end of the article paper concerns specifically the deployment of FD amplifyand-forward (AF) relays, or repeaters, that can satisfy the demand of high data throughput and extend coverage area in modern networks, without increasing frequency reuse $[6,7]$.

The main impediment for FD implementation is the self-interference (SI) caused by the coupling of the transmitted signal to the receiver chain. In compact transceivers, the interference level is usually high compared to the signal of interest [8], depending on the transmitted signal power, the isolation between transmitter and receiver antennas, and the surrounding environmental reflectors. Self-interference is mitigated by a combination of passive and active antenna cancellation $[9,10]$ and RF cancelers [11, 12], both in the analog domain; and a digital cancellation [13-16]. Antenna cancellation techniques aim to reduce the physical coupling between the transmitter and the receiver antennas. Passive techniques 
optimize the distance and the orientation of the antennas, and include absorbers to lower the interference. On the other hand, active techniques are based on the use of multiple antennas and beamforming. After that, RF cancellation subtracts a contribution of the transmitted signal at the receiver for further SI mitigation. Analog stages reduce the SI in order to relax the specifications of the low-noise amplifier and to avoid the desired signal to be drowned by the analog-to-digital converter (ADC) quantization noise [17]. After that, the digital canceler mitigates the residual interference.

Many cancelers have been proposed in the literature $[1,9,15,16]$, but their performance is greatly affected by hardware imperfections. In particular, the effect of power amplifier (PA) non-linearity for FD transceivers is considered in [3,18-21], while the HD relay case is treated in [22]. A widely linear I/Q imbalance and SI cancellation concept is proposed in [14] for a generic FD transceiver and in [23] for an AF FD relay. Additionally, the outage probability of a FD decode-and-forward relay link with I/Q imbalance is derived [24]. On the other hand, the effect of ADC quantization noise is analyzed in [17]. The most harmful RF nonideality is the PA non-linearity that closely depends on the PA input back off (IBO) [3]. The higher the IBO the lower the PA non-linear effect but also the lower the PA efficiency.

Additionally, the IBO (or equivalently the PA transmit power) sets an interesting trade off in the signal-tointerference-plus-noise ratio (SINR) of the two-hop relay link. If the IBO decreases, the signal-to-noise ratio (SNR) at the destination increases, but at the same time this also increases PA non-linear interference level and the SI, reducing the SINR at the relay input. As a consequence, an optimal IBO that maximizes the SINR at destination can be found.

In this work, we derive expressions that bound the optimal IBO that maximizes the SINR at the destination, considering RF impairments. First, we find the expression of the SINR at the destination considering the SI and I/Q imbalance canceler proposed in [23]. Then, we derive lower and upper bounds for the optimal IBO that maximizes the SINR. The SINR maximization can be seen as a suboptimal minimization of the bit error rate (BER). The BER minimization problem was previously solved numerically in [23]. Additionally, we provide closed-form expressions for the bounds considering the soft limiter (SL) PA model, and present numerical solutions for more general PA models. The analysis presented in this article is valid no matter the chosen PA model or its non-linear compensation. Finally, using simulations, we compare the obtained theoretical bounds with the numerical maximization of the SINR and the minimization of the BER to show that our derivations provide a good approximation of the optimal IBO.
The rest of the paper is organized as follows: In Section 2, we introduce the system model and the hardware imperfections. We find the optimal IBO that maximizes the SINR of the relay link in Section 3. In Section 4, we compare the proposed bounds with the numerical maximization of the SINR and the minimization of the BER, for different conditions. Finally, we conclude the paper in Section 5.

\section{Signal model for the two-hop relay link}

We consider a two-hop relaying scheme where the source (S) reaches the destination (D) through a relay (R), without a direct link between the source and the destination. We focus on the downlink, i.e., the link base station-relay-mobile device. The relay operates in the full-duplex mode and implements the amplifyand-forward protocol while signals are modulated with orthogonal frequency-division multiplexing (OFDM). We assume perfect time and frequency synchronization and that the cyclic prefix $(\mathrm{CP})$ is longer than channel impulse responses.

A simplified digital baseband model of an AF relay is illustrated in Fig. 1, where $x(n)$ is the OFDM signal transmitted by the source, $\eta(n)$ is white Gaussian thermal noise at the relay input with variance $\sigma_{\eta}^{2}, u_{r}(n)$ is the received signal distorted by the coupling feedback path while $u_{d c}(n)$ is the corresponding baseband signal, $u_{a d}(n)$ is the digital signal after the ADC, $u_{w}(n)$ is the compensated signal, $u_{d a}(n)$ is the signal after digital-to-analog conversion (DAC), $u_{u c}(n)$ is the RF signal, $y(n)$ is the relay output signal after high-power amplification, and $\eta_{d}(n)$ is white Gaussian thermal noise at the destination with variance $\sigma_{d}^{2}$.

The signal propagates through the two-hop link formed by the source-relay channel $H_{s r}(z)=h_{s r, 0}+h_{s r, 1} z^{-1}+\ldots+$ $h_{s r, L_{s r}} z^{-L_{s r}}$ and the relay-destination channel $H_{r d}(z)=$

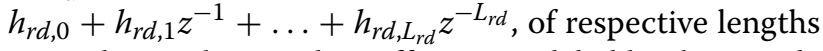
$L_{s r}$ and $L_{r d}$. The coupling effect is modeled by the strictly causal SI channel $H_{s i}(z)=h_{s i, 1} z^{-1}+\ldots+h_{s i, L_{s i}} z^{-L_{s i}}$, of length $L_{s i}$. For the downlink scenario, the source-relay channel is considered static multipath since the source and the relay are both fixed infrastructure-based nodes, and it can be modeled as a static frequency-selective channel. Due to the destination mobility, the relaydestination channel is better modeled as a frequencyselective Rayleigh fading channel. The SI channel can be modeled as Rician due to the strong coupling between the transmitter and receiver antennas. This coupling is significant, because transmitter and receiver antennas are typically close to each other in a FD device, or transmitter and receiver chains may even share the same antenna array. The effect of antenna and RF cancellation stages is to reduce the magnitude of the main path of the SI channel $[1,9,11]$. We assume that the 


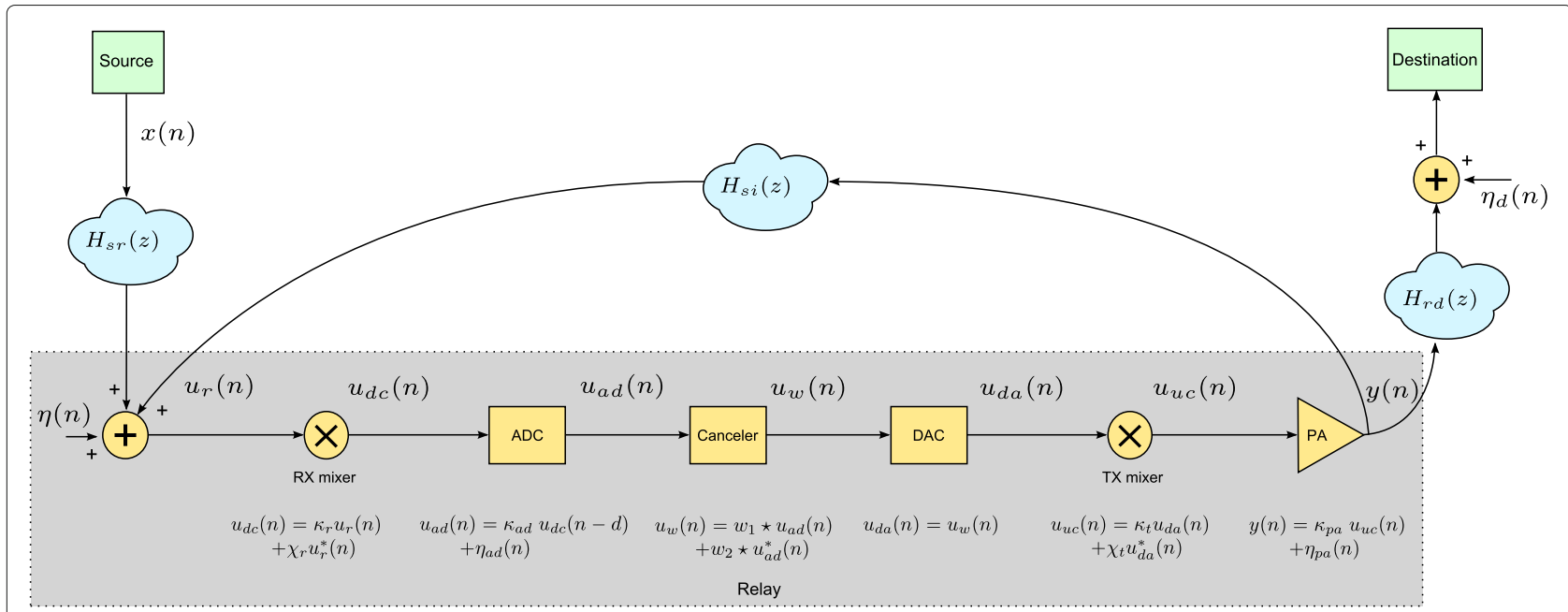

Fig. 1 Two-hop relay link model

residual baseband SI channel already takes into account these cancellation effects.

The proposed widely linear I/Q compensation and SI cancellation scheme consists of a pair of filters: $W_{1}(z)$ and $W_{2}(z)$.

We assume that electronics in the source and destination transceivers are ideal, in order to focus on the effects of relay disturbances on the system performance. In the following, we present imperfection models of the downand up-conversion mixers, the $\mathrm{ADC}$, the $\mathrm{DAC}$, and the PA of the relay. We model all the imperfections in the transceiver front-ends using their digital baseband normalized equivalents. The amplification effects and the antenna and RF attenuation are absorbed by the SNRs at the relay and destination inputs and the channel models.

The effect of the down- and up-conversion mixers over the baseband digital signal can be modeled as

$$
\begin{aligned}
& u_{d c}(n)=\kappa_{r} u_{r}(n)+\chi_{r} u_{r}^{*}(n) \\
& u_{u c}(n)=\kappa_{t} u_{d a}(n)+\chi_{t} u_{d a}^{*}(n)
\end{aligned}
$$

where $\kappa_{r}=\left(1+\alpha_{r} e^{-j \theta_{r}}\right) / 2, \chi_{r}=\left(1-\alpha_{r} e^{j \theta_{r}}\right) / 2, \kappa_{t}=(1+$ $\left.\alpha_{t} e^{-j \theta_{t}}\right) / 2, \chi_{t}=\left(1-\alpha_{t} e^{j \theta_{t}}\right) / 2$; and $\alpha_{r}, \alpha_{t}, \theta_{r}$, and $\theta_{t}$ are respectively the amplitude and phase mismatches of the receiver and transmitter mixers [25].

We recognize that the ADC effects can be modeled by a scaling factor $\kappa_{a d}$ and an additive quantization noise term [17]. The variance of $\eta_{a d}(n)$ can be calculated as [3]

$$
\sigma_{a d}^{2}=\mathrm{E}\left\{\left|u_{d c}\right|^{2}\right\} 10^{-\left(6.02 b+4.76-\mathcal{P}\left(u_{d c}\right)\right) / 10}
$$

where $\mathrm{E}\{\cdot\}$ is the expectation operator, $b$ is the bit resolution of the ADC, and $\mathcal{P}\left(u_{d c}\right)$ is the peak-to-average power ratio of the input signal $u_{d c}(n)$ expressed in decibels. Additionally, we include a processing delay of $d$ samples in the ADC, i.e., $u_{a d}(n)=\kappa_{a d} u_{d c}(n-d)+\eta_{a d}(n)$. The
DAC is regarded ideal, i.e., $u_{d a}(n)=u_{w}(n)$. Considering a single local oscillator at the relay and a short processing delay, as it is the case of AF relays, the phase noise effect can be neglected [26].

If the number of subcarriers is sufficiently large, $x(n)$ becomes Gaussian and the PA output signal can be modeled according to the extended version of the Bussgang theorem [27] as

$$
y(n)=f\left(u_{u c}(n)\right) \simeq \kappa_{p a} u_{u c}(n)+\eta_{p a}(n)
$$

where $f(\cdot)$ is the non-linear response of the PA and $\kappa_{p a}$ with $\left|\kappa_{p a}\right| \leq 1$ is a constant. The first complex coefficient is just a scaling factor over the up-converted signal $u_{d c}(n)$, whereas $\eta_{p a}(n)$ is an additive distortion term. Eq. (4) is able to approximate the behavior of different PA models, from an ideal SL to a PA with nonlinear compensation. When the PA operates sufficiently close to its saturation level, the distortion noise term $\eta_{p a}(n)$ becomes approximately Gaussian. Then, we can calculate the parameters of the equivalent linear model (4) as follows [28]:

$$
\begin{aligned}
\kappa_{p a} & =\frac{\mathrm{E}\left\{u_{u c}^{*}(n) f\left(u_{u c}(n)\right)\right\}}{\mathrm{E}\left\{\left|u_{u c}(n)\right|^{2}\right\}} \\
\sigma_{p a}^{2} & =\mathrm{E}\left\{\left|f\left(u_{u c}(n)\right)\right|^{2}\right\}-\left|\kappa_{p a}\right|^{2} \mathrm{E}\left\{\left|u_{u c}(n)\right|^{2}\right\} .
\end{aligned}
$$

We consider the SL and the solid-state power amplifier (SSPA) models to describe the non-linear PA response. The SL model is defined as

$$
f_{S L}\left(u_{u c}\right)= \begin{cases}\frac{A_{c}}{\nu} u_{u c}(n) & \text { for }\left|u_{u c}(n)\right| \leq \frac{A_{s}}{A_{c}} v \\ A_{s} & \text { for }\left|u_{u c}(n)\right|>\frac{A_{s}}{A_{c}} v\end{cases}
$$

where $A_{s}$ is the PA saturation voltage, $A_{c}=A_{s} / P_{u c}$ is the clipping level, $P_{u c}=\mathrm{E}\left\{\left|u_{u c}(n)\right|^{2}\right\}$ is PA input signal power, 
and $v^{2}$ is the IBO. The solution of (5) for the model in (6) results in

$$
\begin{aligned}
& \kappa_{p a}(v)=\frac{A_{c}}{v}\left(1-\exp \left(-v^{2}\right)+\frac{\sqrt{\pi} v}{2} \operatorname{erfc}(v)\right) \\
& \sigma_{p a}^{2}(v)=\frac{A_{c}}{v^{2}}\left(1-\exp \left(-v^{2}\right)\right)-\kappa_{p a}^{2}(v)
\end{aligned}
$$

Alternatively, the SSPA is defined as

$$
f_{\mathrm{SSPA}}\left(u_{u c}(n)\right)=\frac{\left|u_{u c}(n)\right| / \nu}{\left[1+\left(\left|u_{u c}(n)\right| / \nu A_{s}\right)^{2 p}\right]^{1 / 2 p}} e^{j \angle u_{u c}(n)}
$$

where $p$ is a model parameter that adjusts the smoothness of the transition from the linear region to the saturation region. There is no close-form solution for $\kappa_{p a}(v)$ and $\sigma_{p a}^{2}(v)$ for the SSPA model, so in the simulation section, we obtain these parameters by solving numerically (5).

By denoting the inverse $z$-transform of the widely linear filters $W_{1}(z)$ and $W_{2}(z)$ with $w_{1}(n)$ and $w_{1}(n)$, respectively, the least squares (LS) SI and source-relay (SR) channel canceler output becomes [23]

$$
u_{w}(n)=w_{1}(n) \star u_{a d}(n)+w_{2}(n) \star u_{a d}^{*}(n)
$$

where $\star$ denotes convolution,

$$
\begin{aligned}
& w_{1}(n)=\frac{\kappa_{r}^{*} \kappa_{t}^{*} c(n)+\chi_{t} \kappa_{r}^{*} c^{*}(n)}{\left(\left|\kappa_{r}\right|^{2}-\left|\chi_{r}\right|^{2}\right)\left(\left|\kappa_{t}\right|^{2}-\left|\chi_{t}\right|^{2}\right)} \\
& w_{2}(n)=\frac{-\chi_{r} \kappa_{t}^{*} c(n)-\kappa_{r} \chi_{t} c^{*}(n)}{\left(\left|\kappa_{r}\right|^{2}-\left|\chi_{r}\right|^{2}\right)\left(\left|\kappa_{t}\right|^{2}-\left|\chi_{t}\right|^{2}\right)}
\end{aligned}
$$

and the z-transform of $c(n)$ is given by

$$
C(z)=\frac{1}{\kappa_{a d}\left[H_{s r}(z)+\kappa_{p a} H_{s i}(z) z^{-d}\right]} .
$$

Filter $c(n)$ compensates for the SR and the SI channels. The derivation of the coefficients (11) and (12) is included in Appendix 1.

With the description of all the impairments at hand, we can finally derive the complete input-output relation of the relay. The signal at the relay output is given by

$$
\begin{aligned}
y(n)= & \kappa_{p a}\left[x \star h_{s r}(n-d)+y \star h_{s i}(n-d)+\eta(n-d)\right] \star \Upsilon_{1}(n) \\
& +\kappa_{p a}\left[x \star h_{s r}(n-d)+y \star h_{s i}(n-d)+\eta(n-d)\right]^{*} \star \Upsilon_{2}(n) \\
& +\kappa_{p a} \eta_{a d}(n) \star \Phi_{1}(n)+\kappa_{p a} \eta_{a d}^{*}(n) \star \Phi_{2}(n)+\eta_{p a}(n) .
\end{aligned}
$$

The effects of the ADC, the canceler, and the up- and down-conversion on the input signal can be represented by

$$
\begin{aligned}
\Upsilon_{1}(n)= & \kappa_{a d} \kappa_{t} \kappa_{r} w_{1}(n)+\kappa_{t} \kappa_{a d}^{*} \chi_{r}^{*} w_{2}(n) \\
& +\chi_{t} \kappa_{a d}^{*} \chi_{r}^{*} w_{1}^{*}(n)+\kappa_{a d} \chi_{t} \kappa_{r} w_{2}^{*}(n) \\
\Upsilon_{2}(n)= & \kappa_{a d} \kappa_{t} \chi_{r} w_{1}(n)+\kappa_{t} \kappa_{a d}^{*} \kappa_{r}^{*} w_{2}(n) \\
& +\chi_{t} \kappa_{a d}^{*} \kappa_{r}^{*} w_{1}^{*}(n)+\kappa_{a d} \chi_{t} \chi_{r} w_{2}^{*}(n)
\end{aligned}
$$

whereas the effect of the canceler and the up-conversion on the ADC noise results

$$
\begin{aligned}
& \Phi_{1}(n)=\kappa_{t} w_{1}(n)+\chi_{t} w_{2}^{*}(n) \\
& \Phi_{2}(n)=\kappa_{t} w_{2}(n)+\chi_{t} w_{1}^{*}(n)
\end{aligned}
$$

Now, we are able to write the relay output after I/Q, SI, and SR channel compensation. If we replace filter (12) in (11) and the result in (13), we obtain:

$$
\begin{aligned}
y(n)= & \kappa_{p a} x(n-d)+\kappa_{p a} \Psi_{1} \eta_{a d} \star c^{*}(n)+\kappa_{p a} \Psi_{2} \eta_{a d}^{*} \star c(n) \\
& +\kappa_{p a} \eta(n) \star c(n-d)+\eta_{p a}(n)
\end{aligned}
$$

where

$$
\begin{aligned}
& \Psi_{1}=\frac{\kappa_{r}^{*} \kappa_{t} \chi_{t}-\chi_{r}^{*} \kappa_{t} \chi_{t}}{\left(\left|\kappa_{r}\right|^{2}-\left|\chi_{r}\right|^{2}\right)\left(\left|\kappa_{t}\right|^{2}-\left|\chi_{t}\right|^{2}\right)} \\
& \Psi_{2}=\frac{\left|\chi_{t}\right|^{2} \kappa_{r}-\left|\kappa_{t}\right|^{2} \chi_{r}}{\left(\left|\kappa_{r}\right|^{2}-\left|\chi_{r}\right|^{2}\right)\left(\left|\kappa_{t}\right|^{2}-\left|\chi_{t}\right|^{2}\right)}
\end{aligned}
$$

In the next section, we obtain the SINR at the destination from (17). Then, we find a lower and an upper bound for the optimal IBO that maximizes the SINR.

\section{Optimization of the relay power amplifier input back-off}

The residual interference and RF impairments present at the relay output limit the link performance. Since these terms depend on the relay PA IBO, an optimum value that maximizes the SINR of the complete link can be found. A low IBO setting in the relay PA increases the relay transmission power and leads to a high SNR at the destination but at the same time, it produces large SI reducing SINR at the relay input. Additionally, low IBOs also increase the non-linear behavior of the PA, as described by (5). In contrast, a high IBO limits the SNR at the destination but increases SINR at the relay input. In this section, we first propose an expression of the SINR at the destination considering RF impairments and the SI and I/Q imbalance canceler proposed in [23]. Then, upper and lower bounds for the optimal IBO that maximizes the SINR at the destination are obtained.

The signal at destination, after the relay-destination channel $h_{r d}(n)$, is given by

$$
\begin{aligned}
y_{d}(n)= & \kappa_{p a} x \star h_{r d}(n-d)+\kappa_{p a} \Psi_{1} \eta_{a d} \star c^{*} \star h_{r d}(n) \\
& +\kappa_{p a} \Psi_{2} \eta_{a d}^{*} \star c \star h_{r d}(n)+\kappa_{p a} \eta \star c \star h_{r d}(n-d) \\
& +\eta_{p a} \star h_{r d}(n)+\eta_{d}(n)
\end{aligned}
$$

If an OFDM symbol has length $N_{t}=N+N_{c p}$, where $N$ is the useful data and $N_{c p}$ is the cyclic prefix, the output of the discrete Fourier transform (DFT) at the destination corresponding to one symbol can be written as 


$$
\begin{aligned}
Y_{D}(k)= & \kappa_{p a} X(k) H_{r d}(k) e^{\frac{-j 2 \pi k d}{N}}+\kappa_{p a} \Psi_{1} \gamma_{a d}(k) \\
& +\kappa_{p a} \Psi_{2} \bar{\gamma}_{a d}(k)+\kappa_{p a} \gamma_{\eta}(k) \\
& +\gamma_{p a}(k)+\eta_{d}(k)
\end{aligned}
$$

where $X(k), H_{r d}(k)$, and $\eta_{d}(k)$ are the $N$-DFTs of $x(n), h_{r d}(n)$, and $\eta_{d}(n)$, respectively. Additionally, $\gamma_{a d}(k)=\operatorname{DFT}\left\{\eta_{a d} \star c^{*} \star h_{r d}(n)\right\}, \bar{\gamma}_{a d}(k)=$ $\operatorname{DFT}\left\{\eta_{a d}^{*} \star c \star h_{r d}(n)\right\}, \gamma_{\eta}(k)=\operatorname{DFT}\left\{\eta \star c \star h_{r d}(n-d)\right\}$, and $\gamma_{p a}(k)=\operatorname{DFT}\left\{\eta_{p a} \star h_{r d}(n)\right\}$; for $n$ belonging to the OFDM symbol.

From (21) it is possible to obtain the SINR per subcarrier at the destination as follows:

$\Xi_{d}(k)=\frac{\kappa_{p a}^{2}\left|H_{r d}(k)\right|^{2} P_{x}(k)}{\kappa_{p a}^{2}\left|H_{r d}(k)\right|^{2}\left[P_{a d}(k)+\bar{P}_{a d}(k)+P_{n}(k)\right]+\left|H_{r d}(k)\right|^{2} P_{p a}(k)+P_{d}(k)}$

where $\mathrm{E}\left\{|X(k)|^{2}\right\}=P_{x}(k)$ is the signal power at subcarrier $k$, E $\left\{\left|\Psi_{1} \gamma_{a d}(k)\right|^{2}\right\}=\left|H_{r d}(k)\right|^{2} P_{a d}(k)$ with $P_{a d}(k)=$ $\left|\Psi_{1}\right|^{2} \sigma_{a d}^{2}|C(-k)|^{2}$, and $\mathrm{E}\left\{\left|\Psi_{2} \bar{\gamma}_{a d}(k)\right|^{2}\right\}=\left|H_{r d}(k)\right|^{2} \bar{P}_{a d}(k)$ with $\bar{P}_{a d}(k)=\left|\Psi_{2}\right|^{2} \sigma_{a d}^{2}|C(k)|^{2}$ are the power terms due to the ADC. E $\left\{\left|\gamma_{\eta}(k)\right|^{2}\right\}=\left|H_{r d}(k)\right|^{2} P_{n}(k)$ with $P_{n}(k)=$ $\sigma_{\eta}^{2}|C(k)|^{2}$ is the thermal noise seen at the relay output, $\mathrm{E}\left\{\left|\gamma_{p a}(k)\right|^{2}\right\}=\left|H_{r d}(k)\right|^{2} P_{p a}(k)$ with $P_{p a}(k)=\sigma_{p a}^{2}$ is the interference power related to the PA non-linearity, and $P_{d}(k)=\sigma_{d}^{2}$ is the receiver thermal noise.

In order to find an expression of the optimal IBO $v^{2}$ independent of a particular channel realization, we need to average the effects of $C(k)$ (that depends on $H_{s r}(k)$ and $H_{s i}(k)$, see $\left.(12)\right)$ and $H_{r d}(k)$. Considering first the case of the relay-destination channel, we assume it follows Rayleigh fading, i.e., the terms $s_{k}=\left|H_{r d}(k)\right|^{2}$ have probability density function $p\left(s_{k}\right)=\frac{1}{\sigma_{s_{k}}^{2}} \exp \left(-\frac{s_{k}}{\sigma_{s_{k}}^{2}}\right)$ with $\sigma_{s_{k}}^{2}=\mathrm{E}\left\{\left|H_{r d}(k)\right|^{2}\right\}$. Then, the channel-averaged SINR for all subcarriers can be obtained as

$$
\begin{aligned}
\Xi_{d}= & \mathrm{E}\left\{\sum_{k=0}^{N-1} \frac{\kappa_{p a}^{2} s_{k} P_{x}(k)}{\kappa_{p a}^{2} s_{k}\left[P_{a d}(k)+\bar{P}_{a d}(k)+P_{n}(k)\right]+s_{k} P_{p a}(k)+P_{d}(k)}\right\} \\
= & \sum_{k=0}^{N-1} \frac{\kappa_{p a}^{2} P_{x}(k)}{\sigma_{s_{k}}^{2}\left\{\kappa_{p a}^{2}\left[P_{a d}(k)+\bar{P}_{a d}(k)+P_{n}(k)\right]+P_{p a}(k)\right\}} \\
& \times \int_{0}^{\infty} \frac{s_{k}}{s_{k}+P_{d}(k) /\left\{\kappa_{p a}^{2}\left[P_{a d}(k)+\bar{P}_{a d}(k)+P_{n}(k)\right]+P_{p a}(k)\right\}} \\
& \times \exp \left(-\frac{s_{k}}{\sigma_{s_{k}}^{2}}\right) d s_{k}
\end{aligned}
$$

$$
\begin{aligned}
= & \sum_{k=0}^{N-1} \frac{\kappa_{p a}^{2} P_{x}(k)}{\sigma_{s_{k}}^{2}\left\{\kappa_{p a}^{2}\left[P_{a d}(k)+\bar{P}_{a d}(k)+P_{n}(k)\right]+P_{p a}(k)\right\}} \\
& \times\left[\frac{P_{d}(k) \Gamma(2)}{\kappa_{p a}^{2}\left[P_{a d}(k)+\bar{P}_{a d}(k)+P_{n}(k)\right]+P_{p a}(k)}\right. \\
& \times \exp \left(\frac{P_{d}(k)}{\sigma_{s_{k}}^{2}\left\{\kappa_{p a}^{2}\left[P_{a d}(k)+\bar{P}_{a d}(k)+P_{n}(k)\right]+P_{p a}(k)\right\}}\right) \\
& \left.\times \Gamma\left(-1, \frac{P_{d}(k)}{\sigma_{s_{k}}^{2}\left\{\kappa_{p a}^{2}\left[P_{a d}(k)+\bar{P}_{a d}(k)+P_{n}(k)\right]+P_{p a}(k)\right\}}\right)\right]
\end{aligned}
$$

$$
\begin{aligned}
= & \sum_{k=0}^{N-1} \frac{\kappa_{p a}^{2} P_{x}(k)}{\kappa_{p a}^{2}\left[P_{a d}(k)+\bar{P}_{a d}(k)+P_{n}(k)\right]+P_{p a}(k)} \\
& \times \exp \left(\frac{P_{d}(k)}{\sigma_{s_{k}}^{2}\left\{\kappa_{p a}^{2}\left[P_{a d}(k)+\bar{P}_{a d}(k)+P_{n}(k)\right]+P_{p a}(k)\right\}}\right) \\
& \times \mathcal{E}_{2}\left(\frac{P_{d}(k)}{\sigma_{s_{k}}^{2}\left\{\kappa_{p a}^{2}\left[P_{a d}(k)+\bar{P}_{a d}(k)+P_{n}(k)\right]+P_{p a}(k)\right\}}\right)
\end{aligned}
$$

where $\Gamma(x)$ and $\Gamma(a, x)$ are respectively the Gamma and incomplete Gamma functions. To solve (23) we use $\int_{0}^{\infty} \frac{x^{a-1} e^{-b x}}{x+c} d x=c^{a-1} e^{b c} \Gamma(a) \Gamma(1-a, b c)$ [29, Eq. 3.383.10]; and $\mathcal{E}_{n}(x)=x^{n-1} \Gamma(1-n, x)$ [30, Eq. 5.1.45] in (24) to obtain (25).

Equation 25 can be bounded using the inequation $\frac{1}{x+n}<$ $e^{x} \mathcal{E}_{n}(x) \leq \frac{1}{x+n-1}$ [30, Eq. 5.1.19], as follows:

$$
\begin{aligned}
& \sum_{k=0}^{N-1} \frac{\kappa_{p a}^{2} P_{x}(k)}{\kappa_{p a}^{2}\left[P_{a d}(k)+\bar{P}_{a d}(k)+P_{n}(k)\right]+P_{p a}(k)} \\
& \times\left(\frac{P_{d}(k)}{\sigma_{s_{k}}^{2}\left\{\kappa_{p a}^{2}\left[P_{a d}(k)+\bar{P}_{a d}(k)+P_{n}(k)\right]+P_{p a}(k)\right\}}+2\right)^{-1}
\end{aligned}
$$

$$
\begin{gathered}
<\Xi_{d} \leq \\
\sum_{k=0}^{N-1} \frac{\kappa_{p a}^{2} P_{x}(k)}{\kappa_{p a}^{2}\left[P_{a d}(k)+\bar{P}_{a d}(k)+P_{n}(k)\right]+P_{p a}(k)} \\
\times\left(\frac{P_{d}(k)}{\sigma_{s_{k}}^{2}\left\{\kappa_{p a}^{2}\left[P_{a d}(k)+\bar{P}_{a d}(k)+P_{n}(k)\right]+P_{p a}(k)\right\}}+1\right)^{-1}
\end{gathered}
$$


then,

$$
\begin{gathered}
\sum_{k=0}^{N-1} \frac{\kappa_{p a}^{2} \sigma_{s_{k}}^{2} P_{x}(k)}{P_{d}(k)+2 \sigma_{s_{k}}^{2}\left\{\kappa_{p a}^{2}\left[P_{a d}(k)+\bar{P}_{a d}(k)+P_{n}(k)\right]+P_{p a}(k)\right\}} \\
\sum_{k=0}^{N-1} \frac{\Xi_{d} \leq}{P_{d}(k)+\sigma_{s_{k}}^{2}\left\{\kappa_{s_{k}}^{2} P_{x}\left[P_{a d}(k)+\bar{P}_{a d}(k)+P_{n}(k)\right]+P_{p a}(k)\right\}}
\end{gathered}
$$

It is interesting to note that (29) reduces to the Jensen's inequality for concave functions, i.e., $\mathrm{E}\left\{f\left(s_{k}\right)\right\} \leq f\left(\mathrm{E}\left\{s_{k}\right\}\right)$, where $f\left(s_{k}\right)$ denotes $\Xi_{d}$.

Now, we average the influence of $C(k)$ in the SINR lower (28) and upper (29) bounds. If the number of bits of the ADC is high enough, we can disregard the terms $P_{a d}(k)+\bar{P}_{a d}(k)$. As the actual distribution of $C(k)$ is quite difficult to obtain, we assume $C(k)$ has Gaussian distribution to maintain the derivation tractable. Results in Section 4 support this assumption. Then, $v_{k}=|C(k)|^{2}$ is exponentially distributed with probability density function $p\left(v_{k}\right)=\frac{1}{\sigma_{v_{k}}^{2}} \exp \left(-\frac{v_{k}}{\sigma_{v_{k}}^{2}}\right)$, where $\sigma_{v_{k}}^{2}=\mathrm{E}\left\{|C(k)|^{2}\right\}$. Finally, remembering that $P_{n}(k)=\sigma_{\eta}^{2}|C(k)|^{2}$, the upper bound of the SINR can be expressed as:

$$
\begin{aligned}
\Xi_{d} \leq & \mathrm{E}\left\{\sum_{k=0}^{N-1} \frac{\kappa_{p a}^{2} \sigma_{s_{k}}^{2} P_{x}(k)}{P_{d}(k)+\sigma_{s_{k}}^{2}\left\{\kappa_{p a}^{2} \sigma_{\eta}^{2}|C(k)|^{2}+P_{p a}(k)\right\}}\right\} \\
= & \sum_{k=0}^{N-1} \frac{P_{x}(k)}{\sigma_{\eta}^{2} \sigma_{v_{k}}^{2}} \int_{0}^{\infty} \frac{1}{v_{k}+\left\{P_{d}(k)+P_{p a}(k) \sigma_{s_{k}}^{2}\right\} /\left\{\kappa_{p a}^{2} \sigma_{\eta}^{2} \sigma_{s_{k}}^{2}\right\}} \\
& \times \exp \left(-\frac{v_{k}}{\sigma_{v_{k}}^{2}}\right) d v_{k} \\
= & \sum_{k=0}^{N-1} \frac{P_{x}(k)}{\sigma_{\eta}^{2} \sigma_{v_{k}}^{2}} \exp \left(\frac{P_{d}(k)+P_{p a}(k) \sigma_{s_{k}}^{2}}{\kappa_{p a}^{2} \sigma_{\eta}^{2} \sigma_{s_{k}}^{2}}\right) \Gamma(1) \\
& \times \Gamma\left(0, \frac{P_{d}(k)+P_{p a}(k) \sigma_{s_{k}}^{2}}{\kappa_{p a}^{2} \sigma_{\eta}^{2} \sigma_{s_{k}}^{2}}\right) \\
= & \sum_{k=0}^{N-1} \frac{P_{x}(k)}{\sigma_{\eta}^{2} \sigma_{v_{k}}^{2}} \exp \left(\frac{P_{d}(k)+P_{p a}(k) \sigma_{s_{k}}^{2}}{\kappa_{p a}^{2} \sigma_{\eta}^{2} \sigma_{s_{k}}^{2} \sigma_{v_{k}}^{2}}\right) \\
& \times \mathcal{E}_{1}\left(\frac{P_{d}(k)+P_{p a}(k) \sigma_{s_{k}}^{2}}{\kappa_{p a}^{2} \sigma_{\eta}^{2} \sigma_{s_{k}}^{2} \sigma_{v_{k}}^{2}}\right)
\end{aligned}
$$

where in (31) and (32) we use again $\int_{0}^{\infty} \frac{x^{a-1} e^{-b x}}{x+c} d x=$ $c^{a-1} e^{b c} \Gamma(a) \Gamma(1-a, b c)$ [29, Eq. 3.383.10] and $\mathcal{E}_{n}(x)=$ $x^{n-1} \Gamma(1-n, x)$ [30, Eq. 5.1.45], respectively. Equation 33 can be upper-bounded by $\frac{1}{x+n}<e^{x} \mathcal{E}_{n}(x) \leq \frac{1}{x+n-1}[30$, Eq. 5.1.19], as follows:

$$
\begin{aligned}
\Xi_{d} & \leq \sum_{k=0}^{N-1} \frac{P_{x}(k)}{\sigma_{\eta}^{2} \sigma_{v_{k}}^{2}}\left(\frac{P_{d}(k)+P_{p a}(k) \sigma_{s_{k}}^{2}}{\kappa_{p a}^{2} \sigma_{\eta}^{2} \sigma_{s_{k}}^{2} \sigma_{v_{k}}^{2}}\right)^{-1} \\
& =\sum_{k=0}^{N-1} \frac{\kappa_{p a}^{2} \sigma_{s_{k}}^{2} P_{x}(k)}{P_{d}(k)+P_{p a}(k) \sigma_{s_{k}}^{2}}
\end{aligned}
$$

Similar to the above, the lower bound can be averaged as

$$
\begin{aligned}
\Xi_{d}> & E\left\{\sum_{k=0}^{N-1} \frac{\kappa_{p a}^{2} \sigma_{s_{k}}^{2} P_{x}(k)}{P_{d}(k)+2 \sigma_{s_{k}}^{2}\left\{\kappa_{p a}^{2} \sigma_{\eta}^{2}|C(k)|^{2}+P_{p a}(k)\right\}}\right\} \\
= & \sum_{k=0}^{N-1} \frac{P_{x}(k)}{2 \sigma_{\eta}^{2} \sigma_{v_{k}}^{2}} \int_{0}^{\infty} \frac{1}{v_{k}+\left\{P_{d}(k)+2 P_{p a}(k) \sigma_{s_{k}}^{2}\right\} /\left\{2 \kappa_{p a}^{2} \sigma_{\eta}^{2} \sigma_{s_{k}}^{2}\right\}} \\
& \times \exp \left(-\frac{v_{k}}{\sigma_{v_{k}}^{2}}\right) d v_{k} \\
= & \sum_{k=0}^{N-1} \frac{P_{x}(k)}{2 \sigma_{\eta}^{2} \sigma_{v_{k}}^{2}} \exp \left(\frac{P_{d}(k)+2 P_{p a}(k) \sigma_{s_{k}}^{2}}{2 \kappa_{p a}^{2} \sigma_{\eta}^{2} \sigma_{s_{k}}^{2}}\right) \Gamma(1) \\
& \times \Gamma\left(0, \frac{P_{d}(k)+2 P_{p a}(k) \sigma_{s_{k}}^{2}}{2 \kappa_{p a}^{2} \sigma_{\eta}^{2} \sigma_{s_{k}}^{2}}\right) \\
= & \sum_{k=0}^{N-1} \frac{P_{x}(k)}{2 \sigma_{\eta}^{2} \sigma_{v_{k}}^{2}} \exp \left(\frac{P_{d}(k)+2 P_{p a}(k) \sigma_{s_{k}}^{2}}{2 \kappa_{p a}^{2} \sigma_{\eta}^{2} \sigma_{s_{k}}^{2} \sigma_{v_{k}}^{2}}\right) \\
& \times \mathcal{E}_{1}\left(\frac{P_{d}(k)+2 P_{p a}(k) \sigma_{s_{k}}^{2}}{2 \kappa_{p a}^{2} \sigma_{\eta}^{2} \sigma_{s_{k}}^{2} \sigma_{v_{k}}^{2}}\right) \\
> & \sum_{k=0}^{N-1} \frac{P_{x}(k)}{2 \sigma_{\eta}^{2} \sigma_{v_{k}}^{2}}\left(\frac{P_{d}(k)+2 P_{p a}(k) \sigma_{s_{k}}^{2}}{2 \kappa_{p a}^{2} \sigma_{\eta}^{2} \sigma_{s_{k}}^{2} \sigma_{v_{k}}^{2}}+1\right) \\
= & \sum_{k=0}^{N-1} \frac{\kappa_{p a}^{2} \sigma_{s_{k}}^{2} P_{x}(k)}{P_{d}(k)+2 \sigma_{s_{k}}^{2}\left\{\kappa_{p a}^{2} \sigma_{\eta}^{2} \sigma_{v_{k}}^{2}+P_{p a}(k)\right\}}
\end{aligned}
$$

Assuming that $P_{p a}(k)=P_{p a}$ and $P_{d}(k)=P_{d}$ are white processes, $\sigma_{s_{k}}^{2}=\sigma_{v_{k}}^{2}=1$, and $P_{x}(k)=P_{x}$ for all subcarriers; from (34) and (40) SINR bounds can be simplified to

$$
\frac{N P_{x} \kappa_{p a}^{2}(v)}{P_{d}+2\left\{\kappa_{p a}^{2}(\nu) \sigma_{\eta}^{2}+P_{p a}(v)\right\}}<\Xi_{d} \leq \frac{N P_{x} \kappa_{p a}^{2}(v)}{P_{d}+P_{p a}(v)}
$$

where the dependency of $\kappa_{p a}^{2}$ and $P_{p a}$ on the IBO is included.

To find the bounds of the optimal IBO that maximizes the overall SINR, we differentiate the right-hand side and the left-hand side of (40) and equate them 
to zero. Considering first the case of the lower bound, we have

$$
\begin{aligned}
& \frac{d}{d v}\left(\frac{N P_{x} \kappa_{p a}^{2}(v)}{P_{d}+2\left\{\kappa_{p a}^{2}(v) \sigma_{\eta}^{2}+P_{p a}(v)\right\}}\right) \\
= & \frac{2 N P_{x} \kappa_{p a}(v) \frac{d \kappa_{p a}(v)}{d v}\left(P_{d}+2\left\{\kappa_{p a}^{2}(v) \sigma_{\eta}^{2}+P_{p a}(v)\right\}\right)}{\left(P_{d}+P_{p a}(v)\right)^{2}} \\
& -\frac{2 N P_{x} \kappa_{p a}^{2}(v)\left(2 \sigma_{\eta}^{2} \kappa_{p a}(v) \frac{d \kappa_{p a}(v)}{d v}+\frac{d P_{p a}(v)}{d v}\right)}{\left(P_{d}+P_{p a}(v)\right)^{2}} \\
= & \frac{d \kappa_{p a}(v)}{d v}\left(P_{d}+2 P_{p a}(v)\right)-\kappa_{p a}(v) \frac{d P_{p a}(v)}{d v}=0
\end{aligned}
$$

It is interesting to note that the dependency on $\sigma_{\eta}^{2}$ in (41) is missing. This means that it is not possible to mitigate the effect of the noise at the relay input by varying the PA IBO.

In a similar way for the upper bound, we get

$$
\begin{aligned}
& \frac{d}{d v}\left(\frac{N P_{x} \kappa_{p a}^{2}(v)}{P_{d}+P_{p a}(v)}\right) \\
& =\frac{2 N P_{x} \kappa_{p a}(v) \frac{d \kappa_{p a}(v)}{d v}\left(P_{d}+P_{p a}(v)\right)-N P_{x} \kappa_{p a}^{2}(v) \frac{d P_{p a}(v)}{d v}}{\left(P_{d}+P_{p a}(v)\right)^{2}} \\
& =2 \frac{d \kappa_{p a}(v)}{d v}\left(P_{d}+P_{p a}(v)\right)-\kappa_{p a}(v) \frac{d P_{p a}(v)}{d v}=0
\end{aligned}
$$

If we consider the SL model, it is possible to obtain a closed-form solution to (41) and (42). If we replace

$$
\begin{aligned}
\frac{d \kappa_{p a}(v)}{d v}= & \frac{A_{c}}{v^{2}}\left(-1+\left(v^{2}+1\right) \exp \left(-v^{2}\right)\right) \\
\frac{d P_{p a}(v)}{d v}= & \frac{2 A_{c}^{2}}{v^{3}}\left(-1+\left(v^{2}+1\right) \exp \left(-v^{2}\right)\right) \\
& -2 \kappa_{p a}(v) \frac{d \kappa_{p a}(v)}{d v}
\end{aligned}
$$

in the lower bound (41), we obtain

$$
\begin{aligned}
0= & \frac{A_{c}}{v^{2}}\left(-1+\left(v^{2}+1\right) \exp \left(-v^{2}\right)\right)\left(P_{d}+2 P_{p a}(v)\right) \\
& -\kappa_{p a}(v)\left\{\frac{2 A_{c}^{2}}{v^{3}}\left(-1+\left(v^{2}+1\right) \exp \left(-v^{2}\right)\right)\right. \\
& \left.-2 \kappa_{p a}(v) \frac{A_{c}}{v^{2}}\left(-1+\left(v^{2}+1\right) \exp \left(-v^{2}\right)\right)\right\}
\end{aligned}
$$

$$
\begin{aligned}
& =\left(P_{d}+2 P_{p a}(v)\right)-\kappa_{p a}(v) \frac{A_{c}}{v}+\kappa_{p a}^{2}(v) \\
\frac{\operatorname{erfc}(v)}{v} & =\frac{P_{d}}{A_{c}^{2} \sqrt{\pi}}
\end{aligned}
$$

Similarly, for the upper bound we have

$$
\begin{aligned}
0= & 2 \frac{A_{c}}{v^{2}}\left(-1+\left(v^{2}+1\right) \exp \left(-v^{2}\right)\right)\left(P_{d}+P_{p a}(v)\right) \\
& -\kappa_{p a}(v)\left\{\frac{2 A_{c}^{2}}{v^{3}}\left(-1+\left(v^{2}+1\right) \exp \left(-v^{2}\right)\right)\right. \\
& \left.-2 \kappa_{p a}(v) \frac{A_{c}}{v^{2}}\left(-1+\left(v^{2}+1\right) \exp \left(-v^{2}\right)\right)\right\} \\
= & \left(P_{d}+P_{p a}(v)\right)-\kappa_{p a}(v) \frac{A_{c}}{v}+\kappa_{p a}^{2}(v) \\
\frac{\operatorname{erfc}(v)}{v}= & \frac{2 P_{d}}{A_{c}^{2} \sqrt{\pi}}
\end{aligned}
$$

Finally, if we define the solutions of (47) and (50) as $v_{L B}^{2}$ and $v_{U B}^{2}$, respectively, the optimal IBO for the SL model is bounded by $v_{L B}^{2}<v_{o p}^{2} \leq v_{U B}^{2}$. If the expressions of $\kappa_{p a}(v), P_{p a}(v)$, and their derivatives are not available, (42) and (41) can be solved numerically. In the next section, we use this procedure to find the bound for an SSPA model.

Additionally, we can define the numerical maximization of the SINR and the minimization of the BER of the twohop relay link, as follows

$$
\begin{aligned}
v_{S I N R}^{2} & =\arg \max _{v^{2}}\left\{\operatorname{SINR}_{d}\right\} \\
v_{B E R}^{2} & =\arg \min _{v^{2}}\left\{\mathrm{BER}_{d}\right\}
\end{aligned}
$$

where $\mathrm{SINR}_{d}$ and $\mathrm{BER}_{d}$ are the SINR and the BER at the destination, respectively. Equation 52 is solved numerically in [23] to find the optimal IBO. In the next section, this numerical solution is compared with the bounds (47) and (50).

\section{Numerical results}

In this section, we evaluate the accuracy of the proposed lower and upper bounds (41) and (42) with the numerical maximization of the SINR (51) and the minimization of the BER at destination (52); considering PAs with SL and SSPA models and relay-destination channels with frequency-selective and flat fading.

For the simulation, we use an OFDM signal with $N=$ 1024 subcarriers, a cyclic prefix of $N_{c p}=64$, and 16QAM symbols. Gaussian noise $\eta(n)$ corresponding to a SNR of $20 \mathrm{~dB}$ is added. Practical I/Q imbalance levels are 

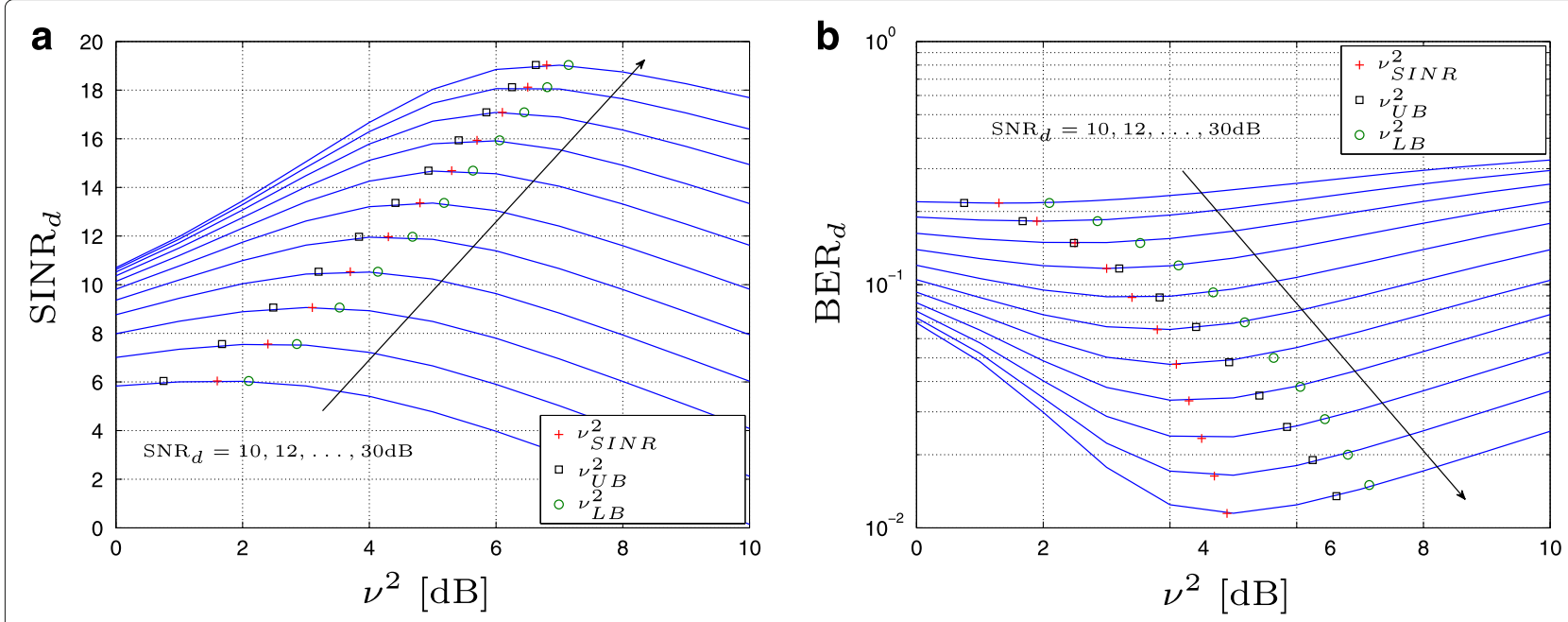

Fig. 2 Maximization of SINR $\mathbf{a}$ and minimization of $B E R \mathbf{b}$ for the $S L$ model and $S N R_{d}=10,12, \ldots, 30 \mathrm{~dB}$. Rayleigh relay-destination channel

considered, i.e., $\alpha^{\mathrm{RX}}=\alpha^{\mathrm{TX}}=1.02, \theta^{\mathrm{RX}}=\theta^{\mathrm{TX}}=1.5^{\circ}$, corresponding to an image rejection ratio (IRR) of $35 \mathrm{~dB}$. We consider an ADC of 12 bits, $\kappa_{a d}=1, \mathcal{P}\left(u_{d c}\right)=13 \mathrm{~dB}$, and a delay of $d=5$. The PA parameters are $A_{s}=1$ for SL and $A_{s}=1, p=2$ for SSPA. The SR channel is a static AWGN channel with $L_{S R}=3$ and the SI channel is a Rician channel with $L_{S I}=3$ and a K-factor ${ }^{1}$ of $5 \mathrm{~dB}$ [1]. We consider two conditions for the relay-destination (RD) link, first a time-varying frequency-selective Rayleigh channel with $L_{R D}=5$ and then a static flat AWGN channel.

We assume that the channels are known. The SNR at the destination is defined as $\mathrm{SNR}_{d}=\mathrm{E}\left\{|x(n)|^{2}\right\} / \sigma_{d}^{2}$ corresponding to an ideal system. In the simulations, we implement the widely linear I/Q imbalance compensation and SI cancellation proposed in [23] and replicated in Appendix 1.

In Fig. 2, we show the result of the numerical SINR maximization (51) and BER minimization (52), and compare them with the derived theoretical bounds (47) and (50), considering a frequency-selective relay-destination channel and $\mathrm{SNR}_{d}=10,12, \ldots, 30 \mathrm{~dB}$. As expected, maximum values lie between the upper and lower bounds. It should be noted that the upper bound underestimates the optimum IBO whereas the lower bound overestimates it. Even when the error in IBO for BER optimization goes from $0.55 \mathrm{~dB}$ for $\mathrm{SNR}_{d}=10$ to $1.73 \mathrm{~dB}$ for $\mathrm{SNR}_{d}=30 \mathrm{~dB}$, the $B E R$ difference between the optimal value and the theoretical bounds is not very large since BER curves are rather flat around the optimum.

In Fig. 3, we plot the result of the numerical maximization of SINR, the upper, and the lower bound as a function of the $\mathrm{SNR}_{d}$, for the same conditions than in Fig. 2. We have that as $\mathrm{SNR}_{d}$ increases the difference between the bounds becomes smaller, giving an accuracy of less than $1 \mathrm{~dB}$ for $\mathrm{SNR}_{d}$ greater than $16 \mathrm{~dB}$.

The difference between the maximization of the SINR and the minimization of the BER is a consequence of the frequency-selectivity of the relay-destination channel. In other words, for a frequency-selective relay-destination channel, the optimization solutions do not coincide. To verify that concept, in Fig. 4 we show the SINR maximization and the BER minimization for a flat fading channel. It is clear that in this case the BER minimization solution also lies between the theoretical bounds.

When closed-form expressions of parameters $\kappa_{p a}(v)$ and $P_{p a}(v)$ are not available, Eqs. (42) and (41) can be solved numerically using the definitions in (5). In Fig. 5 we

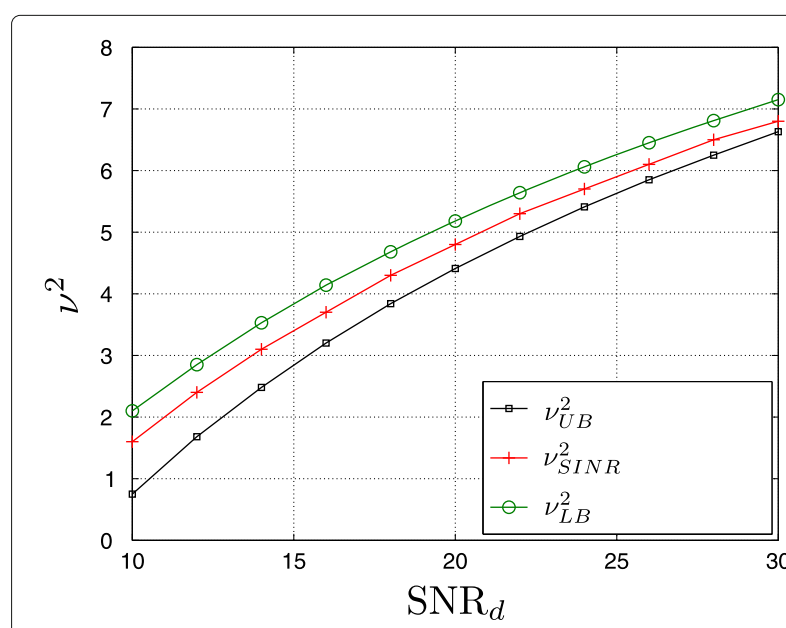

Fig. 3 The result of the numerical maximization of $\operatorname{SINR} v_{S I N R}^{2}$, the upper bound $v_{\mathrm{UB}}^{2}$, and the lower bound $v_{\mathrm{LB}}^{2}$ as a function of the $S N R_{d}$, for the SL model and a Rayleigh relay-destination channel 

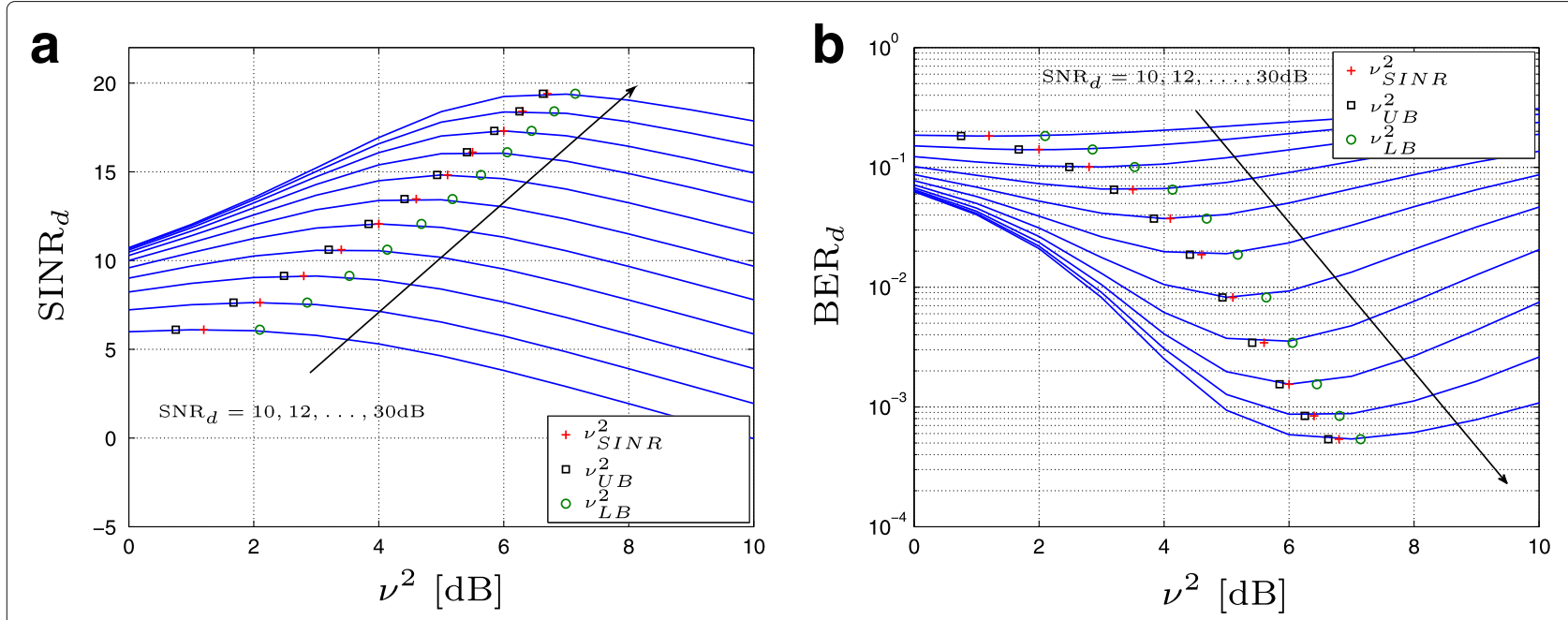

Fig. 4 Maximization of SINR $\mathbf{a}$ and minimization of $B E R \mathbf{b}$ for the $S L$ model and $S N R_{d}=10,12, \ldots, 30 \mathrm{~dB}$. Flat relay-destination channel

show the performance of the upper and the lower bound for the SINR and BER considering the SSPA model. We compare the results with the numerical solution of (51) and (52). As it can be noted, the curves are very similar to those obtained in Fig. 2.

In Fig. 6, we show the BER for the two-hop relay link operating at the optimal point as a function of the $\mathrm{SNR}_{d}$, and compare the performance with fixed IBOs $v^{2}=0,2$, 4,6 , and $8 \mathrm{~dB}$. We include a theoretical and a numerical optimal curve. The theoretical curve is obtained using the upper bound (since it is closer to the optimal IBO), where (50) is solved for each $\mathrm{SNR}_{d}$. The numerical curve is obtained using optimal values found in Fig. 2. Used values for optimal solutions are included in Table 1 . As expected, the numerical curve outperforms the others for every $\mathrm{SNR}_{d}$. The theoretical curve approximates well the numerical solution and performs better than curves with fixed IBOs $v^{2}=0,2,6$, and $8 \mathrm{~dB}$. The curve for $v^{2}=4 \mathrm{~dB}$ has a good performance since the optimal IBO is close to $4 \mathrm{~dB}$ from $\mathrm{SNR}_{d}$ s between 20 and $30 \mathrm{~dB}$. This figure shows that the relay can operate with an optimal PA IBO, minimizing the $\mathrm{BER}_{d}$, if information of $\mathrm{SNR}_{d}$ is available.

\section{Conclusions}

Setting the power amplifier (PA) input back-off (IBO) is a trade-off between the signal-to-noise ratio (SNR)
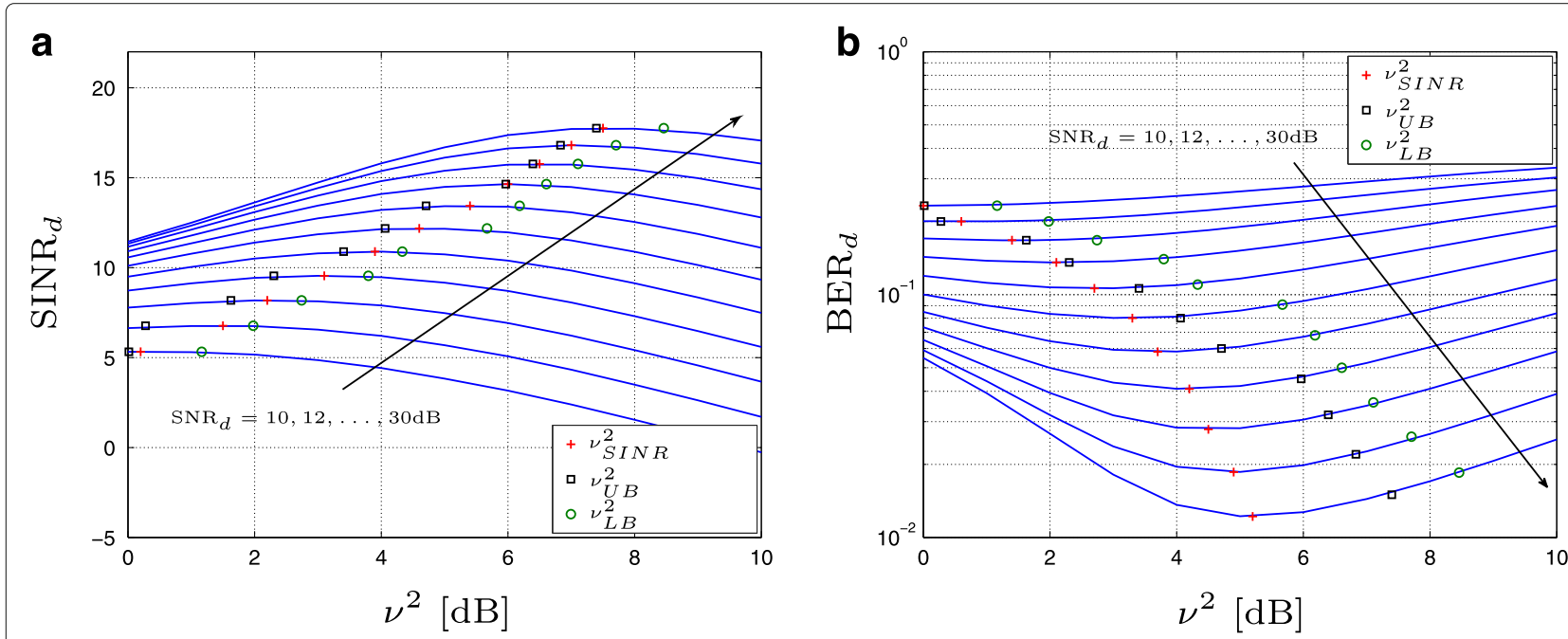

Fig. 5 Minimization of BER for the SSPA model with $p=2, A_{s}=1$, and $S N R_{d}=10,12, \ldots, 30 \mathrm{~dB}$. Rayleigh relay-destination channel 


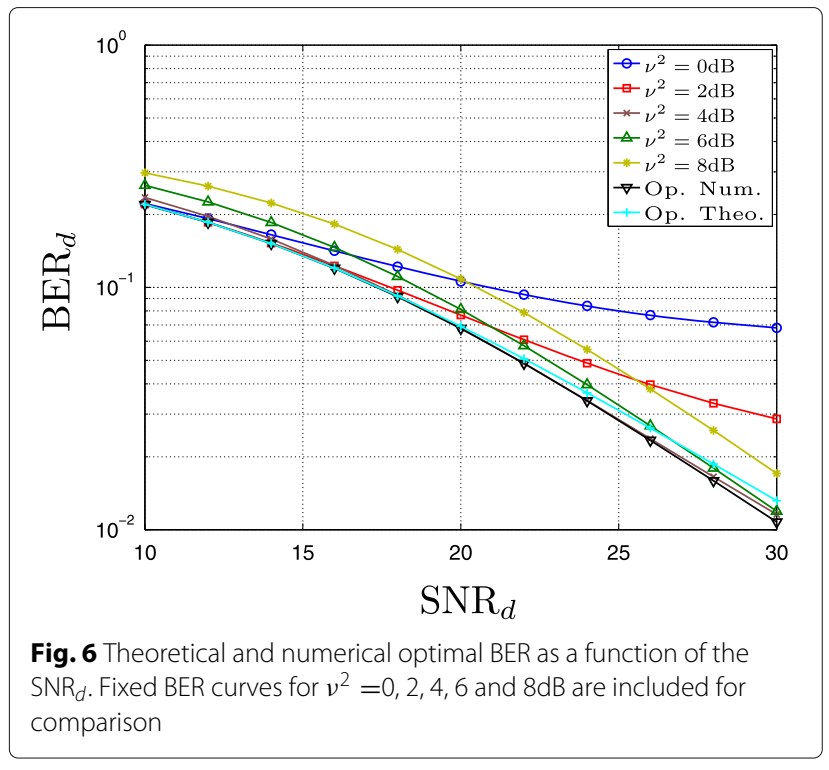

at the destination and the signal-to-interference-plusnoise (SINR) at the relay input. We derived expressions for bounding the optimal IBO that maximizes the SINR at the destination under RF impairments. In particular, we provided closed-form bounds for the soft limiter PA model and numerical solutions for more general models. The bounds proved to be very close to the numerical solution of the optimal SINR minimization. Additionally, we demonstrated that the SNR at the relay input does not affect the IBO optimization. The optimal IBO that minimizes the BER can be bounded by the proposed expressions when the relay-destination channel is flat in frequency and it can be approximated with an acceptable error for frequency-selective channels. Finally, we showed

Table 1 Results of the numerical and theoretical optimal IBOs as a function of the $\mathrm{SNR}_{d}$ used in Fig. 6

\begin{tabular}{lll}
\hline Method & Theoretical & Numerical \\
\hline $\mathrm{SNR}_{d}=10 \mathrm{~dB}$ & $0.75 \mathrm{~dB}$ & $1.30 \mathrm{~dB}$ \\
$\mathrm{SNR}_{d}=12 \mathrm{~dB}$ & $1.67 \mathrm{~dB}$ & $1.90 \mathrm{~dB}$ \\
$\mathrm{SNR}_{d}=14 \mathrm{~dB}$ & $2.48 \mathrm{~dB}$ & $2.50 \mathrm{~dB}$ \\
$\mathrm{SNR}_{d}=16 \mathrm{~dB}$ & $3.20 \mathrm{~dB}$ & $3.00 \mathrm{~dB}$ \\
$\mathrm{SNR}_{d}=18 \mathrm{~dB}$ & $3.84 \mathrm{~dB}$ & $3.40 \mathrm{~dB}$ \\
$\mathrm{SNR}_{d}=20 \mathrm{~dB}$ & $4.41 \mathrm{~dB}$ & $3.80 \mathrm{~dB}$ \\
$\mathrm{SNR}_{d}=22 \mathrm{~dB}$ & $4.93 \mathrm{~dB}$ & $4.10 \mathrm{~dB}$ \\
$\mathrm{SNR}_{d}=24 \mathrm{~dB}$ & $5.41 \mathrm{~dB}$ & $4.30 \mathrm{~dB}$ \\
$\mathrm{SNR}_{d}=26 \mathrm{~dB}$ & $5.85 \mathrm{~dB}$ & $4.50 \mathrm{~dB}$ \\
$\mathrm{SNR}_{d}=28 \mathrm{~dB}$ & $6.25 \mathrm{~dB}$ & $4.70 \mathrm{~dB}$ \\
$\mathrm{SNR}_{d}=30 \mathrm{~dB}$ & $6.63 \mathrm{~dB}$ & $4.90 \mathrm{~dB}$ \\
\hline
\end{tabular}

that the relay can operate with the optimal IBO that minimizes the BER at destination, if information of the second hop SNR is available.

\section{Appendix 1}

\section{Derivation of the coefficients $w_{1}(n)$ and $w_{2}(n)$ of the canceler}

In this section we reproduce for convenience the derivation of zero-forcing (ZF) coefficients $w_{1}(n)$ and $w_{2}(n)$ of the widely linear SI and SR channel canceler, presented in [23]. The coefficients are the result of the combination of three stages that address respectively the I/Q imbalance of the receiver mixer, the SR channel and SI distortion, and the $\mathrm{I} / \mathrm{Q}$ imbalance of the transmitter mixer, as it is depicted in Fig. 7.

The "IQ RX" block is a widely linear post-cancellation filter that is the ZF solution for I/Q imbalance compensation, viz.

$$
u_{R M}(n)=g_{1}^{\mathrm{RX}} \star u_{a d}(n)+g_{2}^{\mathrm{RX}} \star u_{a d}^{*}(n)
$$

where

$$
\begin{aligned}
& g_{1}^{\mathrm{RX}}=\frac{\left(\kappa_{r}\right)^{*}}{\left|\kappa_{r}\right|^{2}-\left|\chi_{r}\right|^{2}} \\
& g_{2}^{\mathrm{RX}}=\frac{-\chi_{r}}{\left|\kappa_{r}\right|^{2}-\left|\chi_{r}\right|^{2}} .
\end{aligned}
$$

The output of SI and SR channel canceler can be written as $u_{C}(n)=u_{R M} \star c(n)$. In a similar way than (53), the output of the widely linear pre-cancellation filter "IQ TX" can be written as

$$
u_{w}(n)=g_{1}^{\mathrm{TX}} \star u_{C}(n)+g_{2}^{\mathrm{TX}} \star u_{C}^{*}(n)
$$

where

$$
\begin{aligned}
& g_{1}^{\mathrm{TX}}=\frac{\left(\kappa_{t}\right)^{*}}{\left|\kappa_{t}\right|^{2}-\left|\chi_{t}\right|^{2}} \\
& g_{2}^{\mathrm{TX}}=\frac{-\chi_{t}}{\left|\kappa_{t}\right|^{2}-\left|\chi_{t}\right|^{2}} .
\end{aligned}
$$

Since there are no noise sources between the filter and the up-conversion mixer, it is able to compensate perfectly the I/Q imbalance at the transmitter.

Finally, we can define the widely linear canceler of Fig. 1 as the combination of the three stages presented above as

$$
\begin{aligned}
& w_{1}(n)=\frac{\left(\kappa_{r}\right)^{*}\left(\kappa_{t}\right)^{*} c(n)+\chi_{t}\left(\kappa_{r}\right)^{*} c^{*}(n)}{\left(\left|\kappa_{r}\right|^{2}-\left|\chi_{r}\right|^{2}\right)\left(\left|\kappa_{t}\right|^{2}-\left|\chi_{t}\right|^{2}\right)} \\
& w_{2}(n)=\frac{-\chi_{r}\left(\kappa_{t}\right)^{*} c(n)-\kappa_{r} \chi_{t} c^{*}(n)}{\left(\left|\kappa_{r}\right|^{2}-\left|\chi_{r}\right|^{2}\right)\left(\left|\kappa_{t}\right|^{2}-\left|\chi_{t}\right|^{2}\right)}
\end{aligned}
$$

according to straightforward substitution. 


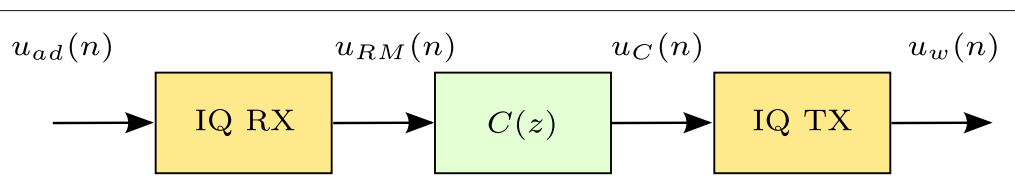

Fig. 7 //Q imbalance compensation and SR-SI equalization

To obtain an expression for $C(z)$, we substitute (57) into (13). Then, we can write the relay output with $\mathrm{I} / \mathrm{Q}$ imbalance compensation as

$$
\begin{aligned}
y(n)= & \kappa_{p a} \kappa_{a d}\left[x \star h_{s r}(n-d)+y \star h_{s i}(n-d)\right] \star c(n) \\
& +\kappa_{p a} \frac{\left[\left(\kappa_{r}\right)^{*} \kappa_{t} \chi_{t}-\left(\chi_{r}\right)^{*} \kappa_{t} \chi_{t}\right]}{\left(\left|\kappa_{r}\right|^{2}-\left|\chi_{r}\right|^{2}\right)\left(\left|\kappa_{t}\right|^{2}-\left|\chi_{t}\right|^{2}\right)} \eta_{a d}(n) \star c^{*}(n) \\
& +\kappa_{p a} \frac{\left[\left|\chi_{t}\right|^{2} \kappa_{r}-\left|\kappa_{t}\right|^{2} \chi_{r}\right]}{\left(\left|\kappa_{r}\right|^{2}-\left|\chi_{r}\right|^{2}\right)\left(\left|\kappa_{t}\right|^{2}-\left|\chi_{t}\right|^{2}\right)} \eta_{a d}^{*}(n) \star c(n) \\
& +\kappa_{p a} \eta(n-d) \star c(n) \\
& +\eta_{p a}(n) .
\end{aligned}
$$

Specifically, (58) shows that the equivalent model of the relay with I/Q imbalance compensation is linear with colored noise. Calculating its $z$-transform and neglecting the noise, we can express the output of the relay as

$$
Y(z)=\frac{\kappa_{p a} \kappa_{a d} H_{s r}(z) C(z) X(z) z^{-d}}{1-\kappa_{p a} \kappa_{a d} H_{s i}(z) C(z) z^{-d}} .
$$

Finally, we can obtain the ZF solution of (58) by equating (59) with $\kappa_{p a} X(z) z^{-d}$. One may note that $C(z)$ does not compensate for the delay or the IBO. The canceler becomes

$$
C(z)=\frac{1}{\kappa_{a d}\left[H_{s r}(z)+\kappa_{p a} H_{s i}(z) z^{-d}\right]} .
$$

Additionally, it is important to make sure that $C(z)$ is a stable filter in order to enable a practical implementation. Considering a simplified scenario with a flat static SR channel $H_{s r}(z)=1$, single tap SI channel $L_{s i}=1$ and $K_{p a}=0.38$ (IBO $=8 \mathrm{~dB}$ and SL PA model). It is easy to show from (60) that the maximum allowable residual SI power, i.e., SI after antenna and RF cancellation, results $1 / K_{p a}^{2}$ larger than the signal of interest. For the SL PA with an $\mathrm{IBO}=8 \mathrm{~dB}$, it equals $\sim 4.3 \mathrm{~dB}$.

\section{Acknowledgements}

Gustavo J. González, Fernando H. Gregorio, and Juan E. Cousseau were funded by the Universidad Nacional del Sur PGI 24/K058 and PGI 24/K059, the ANPCyT PICT-2012-1530, and the CONICET PIP 2012-2014 GI. Taneli Riihonen and Risto Wichman were partly funded by the Academy of Finland under project \# 288249 .

\section{Competing interests}

The authors declare that they have no competing interests.

\section{Publisher's Note}

Springer Nature remains neutral with regard to jurisdictional claims in published maps and institutional affiliations.

\section{Author details}

${ }^{1}$ CONICET-Department of Electrical and Computer Engineering, Universidad Nacional del Sur, Av. Alem 1253, Bahía Blanca, Argentina. ${ }^{2}$ Department of Signal Processing and Acoustics, Aalto University School of Electrical Engineering, Helsinki, Finland.

Received: 16 July 2016 Accepted: 6 April 2017

Published online: 21 April 2017

\section{References}

1. M Duarte, C Dick, A Sabharwal, Experiment-driven characterization of full-duplex wireless systems. IEEE_J_WCOM. 11(12), 4296-4307 (2012)

2. T Riihonen, R Wichman, in 9th International Conference on Cognitive Radio Oriented Wireless Networks and Communications (CROWNCOM). Energy detection in full-duplex cognitive radios under residual self-interference (IEEE, Oulu, 2014), pp. 57-60. doi:10.4108/icst.crowncom.2014.255395

3. D Korpi, T Riihonen, V Syrjälä, L Anttila, M Valkama, R Wichman, Full-duplex transceiver system calculations: Analysis of ADC and linearity challenges. IEEE_J_WCOM. 13(7), 3821-3836 (2014). doi:10.1109/TWC.2014.2315213

4. M Mohammadi, HA Suraweera, Y Cao, I Krikidis, C Tellambura, Full-duplex radio for uplink/downlink wireless access with spatially random nodes. IEEE_J_COM. 63(12), 5250-5266 (2015). doi:10.1109/TCOMM.2015.2495353

5. DWK Ng, Y Wu, R Schober, Power efficient resource allocation for full-duplex radio distributed antenna networks. IEEE_J_WCOM. 15(4), 2896-2911 (2016). doi:10.1109/TWC.2015.2512919

6. A Mattsson, Single frequency networks in DTV. IEEE_J_BC. 51(4), 413-422 (2005)

7. NH Tran, LJ Rodríguez, T Le-Ngoc, Optimal power control and error performance for full-duplex dual-hop AF relaying under residual self-interference. IEEE_J_COML. 19(2), 291-294 (2015). doi:10.1109/LCOMM.2014.2385093

8. J Choi, M Jain, K Srinivasan, P Levis, S Katti, in Proceedings of the 16th Annual International Conference on Mobile Computing and Networking. Achieving single channel, full duplex wireless communication (ACM, |llinois, 2010), pp. 1-12. doi:10.1145/1859995.1859997. http://doi.acm. org/10.1145/1859995.1859997

9. E Everett, A Sahai, A Sabharwal, Passive self-interference suppression for full-duplex infrastructure nodes. IEEE_J_WCOM. 13(2), 680-694 (2014). doi:10.1109/TWC.2013.010214.130226

10. K Haneda, E Kahra, S Wyne, C Icheln, P Vainikainen, in Proceedings of the 4th European Conference on Antennas and Propagation (EUCAP). Measurement of loop-back interference channels for outdoor-to-indoor full-duplex radio relays (IEEE, Barcelona, 2010), pp. 1-5

11. A Raghavan, E Gebara, EM Tentzeris, J Laskar, Analysis and design of an interference canceller for collocated radios. IEEE_J_MTT. 53(11), 3498-3508 (2005)

12. B Debaillie, D-J van den Broek, C Lavin, B van Liempd, EAM Klumperink, C Palacios, J Craninckx, B Nauta, A Pärssinen, Analog/RF solutions enabling compact full-duplex radios. IEEE_J_JSAC. 32(9), 1662-1673 (2014). doi:10.1109/JSAC.2014.2330171

13. T Riihonen, S Werner, R Wichman, Mitigation of loopback self-interference in full-duplex MIMO relays. IEEE_J_SP. 59(12), 5983-5993 (2011)

14. D Korpi, L Anttila, V Syrjälä, M Valkama, Widely linear digital self-interference cancellation in direct-conversion full-duplex transceiver. IEEE_J_JSAC. 32(9), 1674-1687 (2014). doi:10.1109/JSAC.2014.2330093

15. R Lopez-Valcarce, E Antonio-Rodriguez, C Mosquera, F Perez-Gonzalez, 1566-1577. IEEE_J_JSAC. 30(8) (2012). doi:10.1109/JSAC.2012.120923

16. G González, F Gregorio, J Cousseau, in 48th Asilomar Conference on Signals, Systems and Computers. Blind self-interference cancellation for full-duplex relays (IEEE, Pacific Grove, 2014) 
17. T Riihonen, R Wichman, in 46th Asilomar Conference on Signals, Systems and Computers. Analog and digital self-interference cancellation in full-duplex MIMO-OFDM transceivers with limited resolution in A/D conversion (IEEE, Pacific Grove, 2012). doi:10.1109/ACSSC.2012.6488955

18. E Ahmed, AM Eltawil, A Sabharwal, in 47th Asilomar Conference on Signals, Systems and Computers. Self-interference cancellation with nonlinear distortion suppression for full-duplex systems (IEEE, Pacific Grove, 2013), pp. 1199-1203. doi:10.1109/ACSSC.2013.6810483

19. L Anttila, D Korpi, V Syrjälä, M Valkama, in 47 th Asilomar Conference on Signals, Systems and Computers. Cancellation of power amplifier induced nonlinear self-interference in full-duplex transceivers (IEEE, Pacific Grove, 2013), pp. 1193-1198. doi:10.1109/ACSSC.2013.6810482

20. A Masmoudi, T Le-Ngoc, in IEEE International Conference on Communications (ICC). A digital subspace-based self-interference cancellation in full-duplex MIMO transceivers (IEEE, London, 2015), pp. 4954-4959. doi:10.1109/ICC.2015.7249108

21. D Korpi, L Anttila, M Valkama, in IEEE Globecom Workshops. Reference receiver based digital self-interference cancellation in MIMO full-duplex transceivers (IEEE, Austin, 2014), pp. 1001-1007. doi:10.1109/GLOCOMW.2014.7063564

22. T Riihonen, S Werner, F Gregorio, R Wichman, J Hämäläinen, in IEEE Wireless Communication and Networking Conference. BEP analysis of OFDM relay links with nonlinear power amplifiers (IEEE, Sydney, 2010), pp. 1-6. doi:10.1109/WCNC.2010.5506301

23. G González, F Gregorio, J Cousseau, T Riihonen, R Wichman, in European Wireless (EW) conference. Performance analysis of full-duplex AF relaying with transceiver hardware impairments (IEEE, Oulu, 2016)

24. M Awadin, N Al-Dhahir, R Hamila, OFDM full-duplex DF relaying under I/Q imbalance and loopback self-interference. IEEE_J_VT. PP(99), 1-1 (2015). doi:10.1109/TVT.2015.2479257

25. A Tarighat, R Bagheri, AH Sayed, Compensation schemes and performance analysis of IQ imbalances in OFDM receivers. IEEE_J_SP. 53(8), 3257-3268 (2005). doi:10.1109/TSP.2005.851156

26. T Riihonen, $\mathrm{P}$ Mathecken, $\mathrm{R}$ Wichman, in 46 th Asilomar Conference on Signals, Systems and Computers. Effect of oscillator phase noise and processing delay in full-duplex OFDM repeaters (IEEE, Pacific Grove, 2012). doi:10.1109/ACSSC.2012.6489379

27. J Minkoff, The role of AM-to-PM conversion in memoryless nonlinear systems. IEEE_J_COM. 33(2), 139-144 (1985). doi:10.1109/TCOM.1985.1096262

28. D Dardari, V Tralli, A Vaccari, A theoretical characterization of nonlinear distortion effects in OFDM systems. IEEE_J_COM. 48(10), 1755-1764 (2000). doi:10.1109/26.871400

29. IS Gradshteyn, IM Ryzhik, Table of Integrals, Series, and Products, 7th ed. (Academic Press, London, 2007)

30. M Abramowitz, IA Stegun, Handbook of Mathematical Functions with Formulas, Graphs, and Mathematical Tables. (Dover Publications, Washington DC, 1972)

\section{Submit your manuscript to a SpringerOpen ${ }^{\circ}$ journal and benefit from:}

- Convenient online submission

- Rigorous peer review

- Immediate publication on acceptance

- Open access: articles freely available online

- High visibility within the field

- Retaining the copyright to your article

Submit your next manuscript at $\gg$ springeropen.com 